\title{
Epuration naturelle des nitrates des eaux souterraines : possibilités d'application au réaménagement des lacs de gravières
}

\author{
L. Labroue' \\ G. Pinay ${ }^{2}$
}

Mots clés : Nitrates, eaux souterraines, aulnaie, réaménagement, lacs de gravières.

La disparition des nitrates d'une nappe superficielle circulant sous un bois d'aulnes et la mesure directe de l'émission de $\mathrm{N}_{2} \mathrm{O}$ (méthode à l'acétylène) montrent la capacité de ce type de végétation à améliorer la qualité des eaux souterrajnes. L'activité de ce système semble atteindre une efficacité plus élevée que celle des eaux de surface en raison de la conjonction de facteurs favorables : fourniture de carbone organique dégradable, conditions d'anaérobiose, transport de masse des nitrates.

Etant donné l'efficacité du processus, il est suggéré un réaménagement en aulnale des lacs de gravières situés en amont des captages d'eau potable, afin d'en améliorer la qualité des eaux.

Natural nitrate removal from groundwaters. Possibility of application to the reclamation of gravel pit lakes.

Kevwords: Nitrate, groundwaters, alder forest, gravel pit lakes, reclamation.

The disappearance of nitrate from shallow groundwaters circulating beneath an alder forest, along with the direct measurement of $\mathrm{N}_{2} \mathrm{O}$ emission from the soil (acetylen method) show the capacity of this type of vegetation to improve groundwater quality. The activity of the system seems to be more efficient than that observed in surface waters due to the concurrence of favourable factors : a supply of available organic carbon, anaerobic conditions and easy $\mathrm{NO}_{3}^{-} \mathrm{transport}$.

Considering the efficiency of the process, it is suggested that the reclamation of gravel pit lakes up-stream of waterworks with alders could improve the water quality.

\section{Introduction}

Les potentialités d'épuration de l'azote des eaux superficielles ont été mises en évidence depuis long. temps, qu'il s'agisse des lacs (Keeney \& al. 1971) ou des eaux courantes (Owens \& al. 1972). Plus récemment, le rôle des "zones humides " dans l'épuration de la nappe avant son émergence dans les eaux de surface a été signalé par de nombreux auteurs (par exemple, Karr \& Schlosser 1978, Omemik \& al.

1. Laboratoire d'Hydrobiologie, UA 695 C.N.R.S., Université Paul-Sabatier, 118 route de Narbonne, 31062 Toulouse Cédex (France).

2. Centre d'Ecologie des Ressources Renouvelables, C.N.R.S., 29 rue Jeanne Marvig, 31055 Toulouse Cédex (France).
1981, Jacobs \& Gilliam 1985). Enfin, l'éventualité d'une dénitrification dans le sous-sol (Hebert \& al. 1982) ou dans les aquifères eux-mêmes (Bergé 1983 , Mariotti \& Landreau 1985) a soulevé un grand intérêt, en relation avec la contamination croissante des nappes utilisées pour l'alimentation en eau potable.

Cependant, plusieurs points restent à préciser :

- la dénitrification est-elle le processus essentiel dans l'abaissement des concentrations en nitrates observé ?

- si oui, s'agit-il d'un mécanisme purement chimique, biologique ou mixte?

- quelle est l'importance quantitative du phénomène et son intérêt pratique ? 
Nous nous sommes penchés sur ces questions lors de l'étude du devenir d'une nappe circulant sous une forêt alluviale, dans la région toulousaine.

\section{Matériel et méthodes}

Le site étudié (fig. 1) est un bois d'aulnes ayant colonisé un bras mort de la Louge, petit affluent de la Garonne dans laquelle il se jette au niveau de la ville de Muret (Feuille 1/50 000, XX/44:X $=127,6$, Y 5 16,4). La nappe circule dans le sens indiqué sur la figure et traverse un lac de gravière avant de passer sous le bois.
Deux séries de piézomètres ont été mises en placs dans le secteur : la série ABCDEFG dans le bois, par le Centre d'Ecologie des Ressources Renouvelables, à l'automne 1984 ; la série $P$, autour de la gravière, par le Laboratoire régional des Ponts et Chaussées au cours de l'année 1985.

Différents paramètres de l'eau $(\mathrm{pH}$, potenticl redox, conductivité, T.A.C., Carbone dissous, $\mathrm{NO}_{3}$, $\mathrm{NO}_{2}^{-}, \mathrm{NH}_{4}^{+}, \mathrm{Fe}^{++}, \mathrm{Mn}^{++}$, alcalins et alcalinoterreux) et des sols ont été analysés tous les mois. De plus, des mesures de dégagement de $\mathrm{N}_{2} \mathrm{O}$, apres blucage de la dénitrification à ce stade par l'acétylène, ont été effectuées sous cloche et sur carottes de sol ou de sédiments.

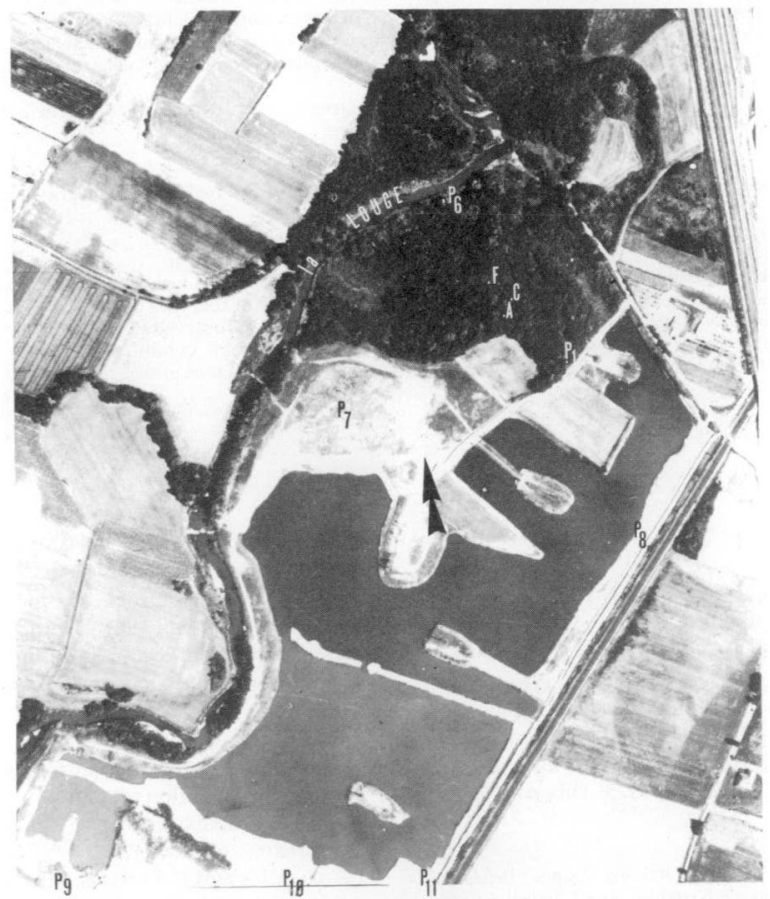

Fig. 1 : Localisation de la zone étudiée. Emplacement des piézomètres. Echelle 1/5 750. 
Les résultats analytiques utilisés dans cet article ont été obtenus de la manière suivante :

- le carbone organique dissous a été dosé sur un appareil Dohrmann Techmation C.T. au Laboratoire d'Ecologie des eaux douces de Lyon I,

- l'azote nitrique par colorimétrie des nitrites après réduction sur colonne cadmium-cuivre (Keeney \& Nelson 1982),

- le fer ferreux par colorimétrie à l'orthophénanthroline (Golterman et al. 1978),

- le manganèse par spectrométrie d'absorption atomique.

\section{Résultats}

L'influence améliorante du lac de gravière sur la teneur en nitrates de la nappe (Tableau I) n'est pas négligeable et confirme les observations de Bergé (1983) et de Donville (1986). La nappe contient 20 à $30 \mathrm{ppm}$ de nitrates en amont $\left(\mathbf{P}_{9,}, \mathbf{P}_{10}, \mathbf{P}_{11}\right)$ contre 2 à $10 \mathrm{ppm}$ en aval $\left(\mathbf{P}_{1}, \mathbf{P}_{7}\right)$. Les teneurs de la gravière clle-même varient entre 2.5 et $15 \mathrm{ppm}$.

Tableau I. - Teneurs en azote nitrique de la nappe aux alentours du site étudié (mg. $\mathrm{t}^{-1}$ ). Hiver 1985-1986.

\begin{tabular}{lccccccc}
\hline & $\mathbf{P}_{1}$ & $\mathbf{P}_{6}$ & $\mathbf{P}_{7}$ & $\mathbf{P}_{8}$ & $\mathbf{P}_{9}$ & $\mathbf{P}_{10}$ & $\mathbf{P}_{11}$ \\
\hline Décembre & 0,63 & $\mathbf{0 , 0 1}$ & - & $\mathbf{1 , 6 0}$ & 0,75 & 5,70 & 6,79 \\
Janvier & $\mathbf{0 , 7 1}$ & $\mathbf{0 , 4 7}$ & - & $\mathbf{1 , 2 7}$ & $\mathbf{2 , 0 0}$ & $\mathbf{4 , 5 2}$ & $\mathbf{4 , 8 2}$ \\
Février & $\mathbf{1 , 3 9}$ & $\mathbf{0 , 3 0}$ & 0,31 & 2,40 & 5,04 & 5,15 & 5,56 \\
Mars & $\mathbf{2 , 1 6}$ & $\mathbf{0 , 0 4}$ & 0,54 & 4,68 & 5,58 & 4,98 & 4,45 \\
\hline
\end{tabular}

Cependant, les performances du bois d'aulnes sont supérieures puisque quelques mètres de trajet suffisent pour faire disparaître les nitrates présents (fig. 2). La décroissance des nit rates s'accompagne de la mise en solution de fer ferreux, de manganèse ainsi que d'un peu d'azote ammoniacal. En été, la disparition des nitrates est effective dès la station $\mathrm{A}$ et on note une accumulation plus importante des éléments réduits et du carbone (C.O.D.).

Plusieurs causes peuvent entraîner une baisse de la concentration en nitrates. Les principales sont la dilution, l'absorption racinaire et la dénitrification. La dilution par le sous-écoulement de la Louge ne peut être invoquée car cette dernière contient de 3 à $17 \mathrm{ppm}$ de nitrates et elle est à l'origine des valeurs élevées observées sur le piezomètre $P_{t}$ en période de crue.
L'absorption racinaire n'est effective que pendant la saison végétative alors que l'épuration fonctionne mème en hiver. Par contre, la dénitrification a été mise en évidence tout au long de l'année par des mesures directes. Elle varie en fonction des sites et des saisons et peut dépasser, dans les conditions optimales, $3 \mathrm{mg}$. dm ${ }^{-3} \cdot \mathrm{j}^{-1}$ (Pinay \& Labroue 1986).

Les conditions favorables à la dénitrification hétérotrophique sont les suivantes: faible tension en oxygène (potentiel redox $<340 \mathrm{mV}$ ), présence de carbone assimilable, d'une microflore dénitrifiante et, bien entendu, présence de nitrates. Ces conditions peuvent être réunies dans les eaux superficielles et les eaux souterraines dont le cycle de l'azote peut être représenté selon les deux modèles conceptuels de la figure 3.

Le modèle eaux superficielles, auquel appartient le lac de gravière, a son activité limitée par la diffusion des nitrates dans la zone anoxique, ce qui donne une réaction d'épuration d'ordre 0,5 (Klapwijk \& Snodgrass 1982). Dans le modèle eaux souterraines, où l'apport de nitrates se fait par transport de masse, c'est d'abord le carbone énergétique qui est le facteur limitant, jusqu'à épuisement des nitrates. Dans notre cas, la diffusion du carbone dissous paraît efficace puisque l'on n'observe pratiquement pas de stratification dans la nappe, il est vrai peu épaisse $(3 \mathrm{~m})$.

La quantification des différents flux dans les deux modèles reste à préciser.

\section{Discussion}

L'efficacité de l'aulnaie dans l'épuration de la nappe est démontrée par le bilan entrée-sortie : en Mars, $10 \mathrm{ppm}$ de $\mathrm{NO}_{3}^{-}$sont réduits en moins de 10 mètres. La mesure directe des dégagements gazeux montre qu'il s'agit essentiellement de dénitrification.

Une telle efficacité peut être liée au fait que la décomposition des litières s'effectue souvent en anaérobiose, ce qui libère des composés organiques solubles susceptibles de migrer dans la nappe. Ils sont oxydés en présence de nitrates (respiration nitrate) ou s'accumulent lorsque ceux-ci sont épuisés (fig. 2). Une application de ce phénomène à l'épuration des aquifères en amont des captages peut être envisagée lorsqu'il existe des gravières. Leur réaménagement en aulnaie pourrait être tenté en prenant 

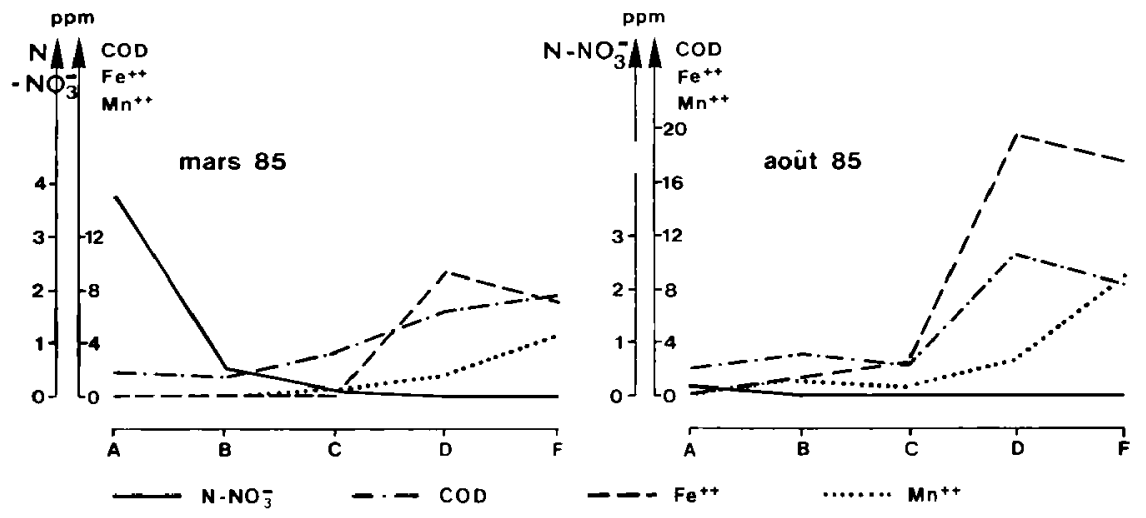

Fig. 2 : Evolution des caractéristiques de la nappe le long d'un transect sous la forêt alluviale (C.O.D = carbone organique dissous).
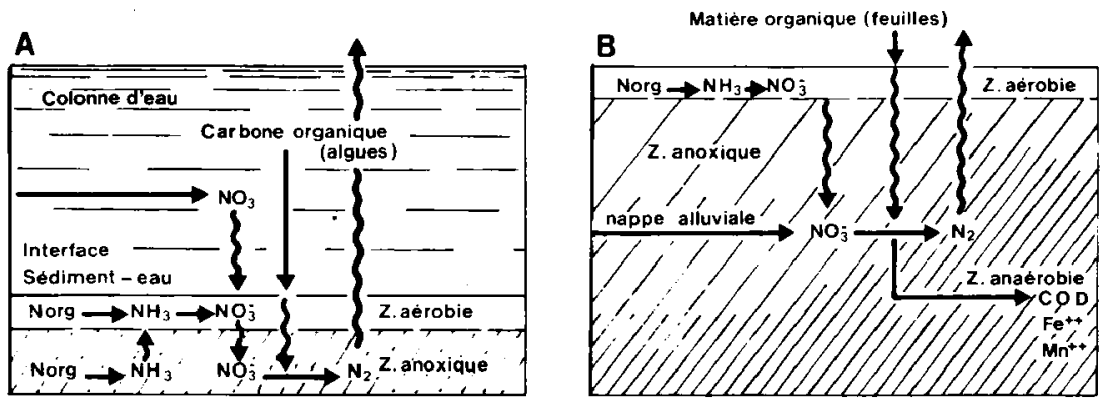

Fig. 3 : Modèles conceptuels du cycle de l'azote dans le cas des eaux superficielles (A) et dans le cas des eaux souterraines (B). 

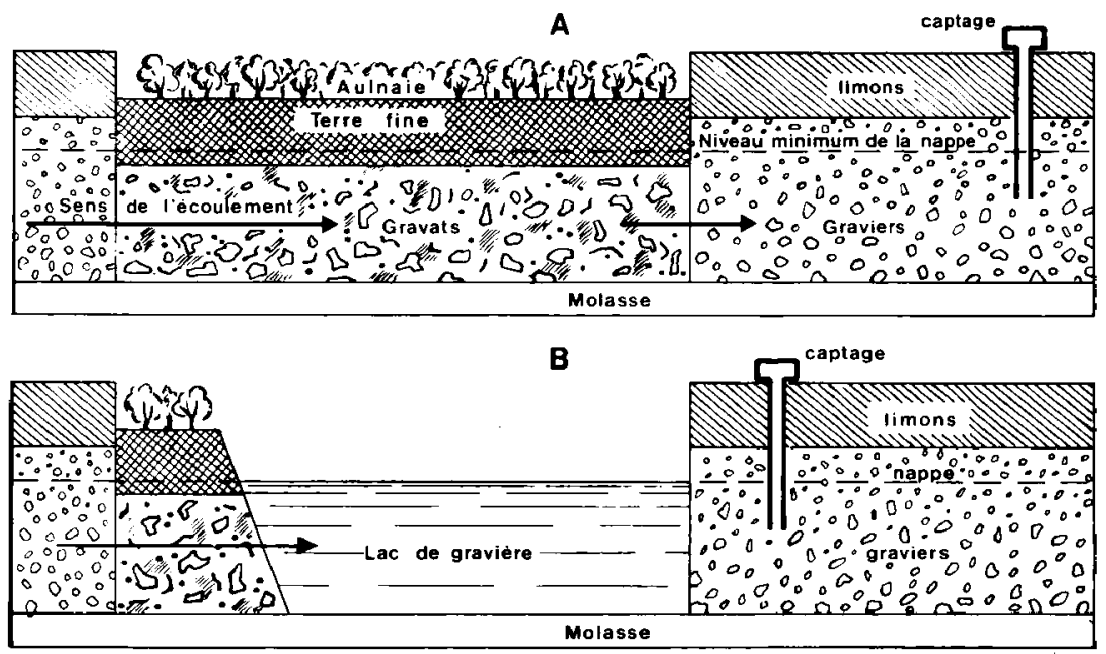

Fig. 4 : Possibilités de réaménagement des lacs de gravières en vue d'une dénitrification de la nappe en amont des captages.

soin d'ajuster le niveau du sol en fonction de la surface piézométrique de manière à obtenir des conditions d'anaérobiose superficielle. La couche de limons favoriserait l'obtention de ces conditions.

Dans le cas de l'aménagement A (comblement total), on peut craindre la sortie d'éléments réducteurs $\left(\mathrm{Fe}^{++}, \mathrm{Mm}^{++}\right.$, C.O.D., $\left.\mathrm{NH}_{4}^{+}\right)$, préjudiciable à la qualité des eaux au niveau du captage. Il faudrait donc évaluer la distance permettant une évolution ou une dilution adéquates. Dans le cas de l'aménagement B (lac + aulnaje), ces inconvénients seraient éliminés, mais les risques de colmatage, inhérents aux plans d'eau, pourraient limiter l'efficacité du procédé.

\section{Conclusions}

L'activité épuratrice de l'aulnaie vis-à-vis des nitrates des nappes alluviales semble liée à une forte activité dénitrifiante hétérotrophe. Celle-ci serait alimentée par la diffusion de carbone soluble, fourni par la fermentation des litières, dans l'épaisseur de l'aquifère.

Ce résultat suggère une application dans le réaménagement des lacs de gravières situées en amont de captages d'eau potable. Cependant, seule la suite des recherches permettra de vérifier si les inconvénients envisagés peuvent être surmontés et s'il s'agit bien d'une solution, à priori élégante, à un double problème.

\section{Remerciements}

Nous remercions vivement C. Arlès et J. Dadour, du Centre d'Ecologie des Ressources Renouvelables C.N.R.S., pour leur collaboration fort appréciée.

Travaux cités

Ber'ge (Ph.). 1983. - Detec tion de l'origine des pollutions azotees d'un aquifère alluvial de la vallée de la Garonne par traçage isotopique. Thèse $3^{*}$ cycle, Ecologie, Toulouse: $221 \mathrm{p}$. 
Donville (B.). 1986. - Evolution des teneurs en nitrates dans les lacs de gravières. Effets sur les eaux souterraines dans la Haute-Garonne (31). Rappont Conseil Général de la HauteGaronne, 84 p., cartes.

Golterman (H.L.), Clymo (R.S.) \& Ohnstad (M.A.M.). 1978. Methods for physical and chemical analysis of fresh waters. I.B.P. Handbook $n^{\circ}$ 8: 213 p.

Heber (J.), Machet (J.M.) \& Remy (J.C.). 1982. - Un cas de diminution de la teneur en nitrates des eaux de percolation par dénitrification non provoquée. XVlle Journées de l'Hydraulique. Nantes. Rapport II-7: 1-7.

Jacobs (T.C.) \& Gilliam (J.W.). 1985. - Riparian losses of nitrate from agricultural drainage waters. I. Environ. Qual., 14 (4) : 472.478.

Karr (J.R.) \& Schlosser (1.J.). 1978. - Water resources and the landwater interface. Science, $201: 229-234$.

Keeney (D.R.), Chen (R.L.) \& Graetz (D.A.). 1971. - Denitrification and nitrate reduction in sediments : Importance to the nitrogen budget of lakes. Nature, $233: 66-67$.

Keeney (D.R.) \& Nelson (D.W.). 1982. - Nitrogen : inorganic forms. In Methods of Soil Artalysis. Part 2, 2nd edition. Page (A.L.), Miller (R.H.), Keeney (D.R.) eds Madison-Wisconsin U.S.A.: 643-698.
Klapwisk (A.) \& Snodgrass (W.S.). 1982. - Experimental measu. rement of sediment nit rification and denitrification in Hamil. ton Harbour, Canada. In Developments in Hydrobiology. Sedi. ment/Freshwater Interaction. SLY (P.G.) ed. Junk Publishers. The Hague, Netherlands: 207-216.

Mariotti (A.) \& Landreau (A.). 1985. - Etude du transfert et de l'évolution des nitrates dans les aquiferes au moyen du traçage iso topique naturel ${ }^{15} \mathrm{~N}$. Site $85 \mathrm{Nitrates}$ dans les eaux. Paris.

Omernik (J.M.), Abernathy (A.R.) \& Male (L.M.). 1981. - Stream nutrient levels and proximity of agricultural and forest land to streams : some relationships. Journal of Soil and Water Con. servation, $36,(4): 227.231$.

Owens (M.), Garland (J.H.N.), Hart (J.C.) et Waad (G.). 1972. Nut rient budgets in rivers. Symp. Zool. Soc. Lond, $29: 21.40$.

Pinay (G.) \& Labroue (L.). 1986. - Une station d'épuration natu. relle des nitrates transportés par les nappes alluviales: I'aul. naie glutineuse. C.R. Acad. Sciences Paris, (sous presse). 